\title{
Dermatopolimiositis en paciente adolescente. Reporte de caso
}

\author{
Dermatopolymyositis in adolescent patient. Case report \\ Scarleth Paola Vásquez Reconco*, Marlon Alexander Cerna Márquez**, \\ Larissa Maydelin Contreras Martínez ${ }^{* *}$, Allan Iván Izaguirre González ${ }^{* * *}$, Cándido Mejía****
}

\section{RESUMEN}

La Dermatopolimiositis (DPM) pertenece a las miopatías inflamatorias idiopáticas (MII), un grupo heterogéneo de miopatías autoinmunitarias sistémicas crónicas, asociadas con una alta morbilidad y discapacidad funcional. Comprende aquellas entidades de naturaleza adquirida que cursan con debilidad muscular y presentan de forma característica un infiltrado inflamatorio y necrosis celular en el tejido muscular estriado. Es una enfermedad rara, con una incidencia global de 2-10 casos por millón de habitantes/año. Presentamos el caso de adolescente masculino de 14 años con antecedente de dermatomiositis, el cual presenta debilidad muscular proximal progresiva, acompañado de mialgias intensas e incapacitantes, presencia de eritema en heliotropo y pápulas de Gottron. Estudios laboratoriales que evidenciaron anemia, alteraciones enzimáticas, reactantes de fase aguda alterados, estudio electromiográfico que evidenció la presencia de polimiositis reactiva, y biopsia de tejido muscular que reportó cambios compatibles con DPM. El diagnóstico de miopatías inflamatorias se sospecha sobre la base de un conjunto de signos y síntomas y es confirmado mediante estudios complementarios, entre los que se incluyen: elevación de enzimas musculares, presencia de autoanticuerpos específicos de miositis,

\footnotetext{
*Médico Residente de segundo año de Pediatría UNAH-VS.

**Médico Residente de tercer año de Pediatría UNAH-VS.

***Doctor en Medicina y Cirugía - UNAH, Asesor de la Federación Internacional de Asociaciones de Estudiantes de Medicina (IFMSA) - Honduras.

****Dermatólogo en HNMCR.

Dirigir correspondecia a: scarlethvasquez 90@hotmail.com

Recibido: 10 de Diciembre 2018 Aceptado: 12 de Agosto 2019
}

electromiografía con patrón miopático, hallazgos específicos en la biopsia. La PDM en niños tiene un comportamiento clínico diferente al adulto por la presencia vasculitis, existiendo varios desordenes que pueden confundir esta entidad y retardar su diagnóstico y tratamiento, por lo tanto, es muy importante el conocimiento de esta enfermedad en la edad pediátrica y establecer comparaciones con lo reportado en la literatura mundial.

\section{PALABRAS CLAVE}

Polimiositis-Dermatomiositis, Polimiositis, Dermatomiositis, Anticuerpos, Debilidad Muscular, Autoanticuerpos, (DesC BVS).

\section{ABSTRACT}

Dermatopolimiositis (MPD) belongs to idiopathic inflammatory myopathies (MII), a heterogeneous group of chronic systemic autoimmune myopathies associated with high morbidity and functional disability. It includes those entities of an acquired nature that present with muscular weakness and characteristically present an inflammatory infiltrate and cell necrosis in the striated muscular tissue. It is a rare disease, with an overall incidence of 2-10 cases per million inhabitants / year. We present the case of a 14-year-old male adolescent with a history of dermatomyositis, which presents progressive proximal muscle weakness, accompanied by intense and disabling myalgias, presence of heliotrope erythema and Gottron papules. Laboratorial studies that showed anemia, enzymatic alterations, altered acute phase reactants, electromyographic study that showed the presence of reactive polymyositis, and muscle tissue 
biopsy that reported changes compatible with MPD. The diagnosis of inflammatory myopathies is suspected on the basis of a set of signs and symptoms and is confirmed by complementary studies, including: elevation of muscle enzymes, presence of myositis-specific autoantibodies, electromyography with myopathic pattern, specific findings in the biopsy. The PDM in children has a different clinical behavior than the adult due to the presence of vasculitis, there are several disorders that can confuse this entity and delay its diagnosis and treatment, therefore, it is very important to know this disease in the pediatric age and establish comparisons with what is reported in world literature.

\section{KEYWORDS}

Polymyositis-Dermatomyositis, Polymyositis, Dermatomyositis, Antobodies, Muscle Weakness, Autoantibodies, (MeSH NLM).

\section{INTRODUCCIÓN}

La dermatomiositis (DM) y la polimiositis (PM) son parte de las miopatías inflamatorias idiopáticas (MII), un grupo heterogéneo de miopatías autoinmunitarias sistémicas crónicas, asociadas con una alta morbilidad y discapacidad funcional.(1) Comprende aquellas entidades de naturaleza adquirida que cursan con debilidad muscular y presentan de forma característica un infiltrado inflamatorio y necrosis celular en el tejido muscular estriado. ${ }^{(2)}$

Es una enfermedad rara, la incidencia global de las miopatías inflamatorias se reporta en rangos de 2-10 casos por millón de habitantes por año. Se han encontrado reportes de hasta 4,9 a 8,4 casos/millón asociados a mayor sospecha clínica y mejoría de técnicas diagnósticas más que a un incremento real de la incidencia misma. La prevalencia se estima en 8/100.000 habitantes. ${ }^{(3)}$ Afecta a personas de todas las edades, con un pico de incidencia en niños de alrededor de 10 años de edad y otro en adultos alrededor de los 40. La razón global de la incidencia mujer: hombre es 2,5:1. Siendo cercana a 1:1 en la niñez y en los casos de asociación a neoplasia, y tan alto como 10:1 cuando coexiste con otra enfermedad del tejido conectivo. Afecta de 3-4 veces más la raza blanca. $^{(4)}$

La enfermedad fue descrita desde 1886 por clínicos alemanes, pero quien acuñó el término de polimiositis fue $\mathrm{E}$. Wagner en 1886 y el de dermatomiositis fue $\mathrm{H}$. Unverricht en 1891; una asociación con neoplasias se publicó en 1916, pero la asociación causal se describió en 1935 respectivamente. Se han producido muchas clasificaciones de la enfermedad y criterios de clasificación, pero las que más se utilizan en la práctica clínica son las propuestas por Bohan y Peter:

- Grupo I: polimiositis primaria idiopática.

- Grupo II: dermatomiositis primaria idiopática.

- Grupo III: dermatomiositis o polimiositis asociadas con neoplasia.

- Grupo IV: dermatomiositis o polimiositis infantil asociadas con vasculitis.

- Grupo V: polimiositis o dermatomiositis asociadas con enfermedades del tejido conjuntivo (síndrome de traslape). ${ }^{(5)}$

\section{CASO CLÍNICO}

Adolescente masculino de 14 años de edad procedente de la zona norte de San Pedro Sula, Honduras, con antecedente de dermatopoliomiositis diagnosticado hace dos años, tratado por consulta externa de dermatología con esteroides orales, (prednisona), hidroxicloroquina (plaquinol), inhibidores de bomba de protones (omeprazol) e inmunomoduladores (metotrexate). Se indicó a paciente en visita previa el omitir tratamiento con plaquinol, presentando posteriormente debilidad proximal (miembros superiores), de forma simétrica, bilateral, 
acompañado de mialgias intensas e incapacitantes para realizar actividades de rutina, acompañado de hiperqueratosis y perdida de la sensibilidad en miembros superiores e inferiores, asimismo presenta dolor abdominal de 4 meses de evolución en epigástrico, refiere que ingiere su tratamiento sin presentar mejoría, por lo que acude al servicio de emergencias pediátricas en donde se decide su ingreso por recaída de cuadro previo.

Al examen físico: paciente en su segunda década de vida, con fascie de enfermedad aguda, vistiendo ropa extrahospitalaria. Con signos vitales: PA: 95/75mmHg, FC: $79 x^{\prime}$, FR: $20 x^{\prime}$, T: $37^{\circ} \mathrm{C}$ Sat O2: $99 \%$. Antropometría: Peso: $45 \mathrm{~kg}$, talla: $1.57 \mathrm{mts}$. Neurológico: Glasgow 15, pupilas isocóricas, normorreactivas, fuerza 4/5 miembros superiores, $3 / 5$ en miembros inferiores, Reflejos ++,
Hemodinámico: hidratado, no signos de bajo gasto, corazón con ritmo regular no soplos, pulsos palpables, llenado capilar menor de 2 seg. Respiratorio: patrón respiratorio regular, pulmones ventilados, no se auscultan ruidos patológicos. Gastrointestinal: abdomen no distendido, RI +, blando, depresible, no masas, no visceromegalia, no dolor a la palpación. Genitourinario: genitales masculinos sin alteración, no lesiones.

Extremidades: simétricas, no edema, con dificultad para la extensión de ambos brazos, con dolor a la movilización en miembros inferiores. Piel y faneras: con eritema heliotropo en cara en región perinasal y miembros inferiores, con engrosamiento de la piel, presencia de pápulas de Gottron en ambas articulaciones metacarpofalángicas bilaterales. (Ver Figura 1).

FIGURA No. 1: A) Eritema heliotropo en cara, región perinasal y miembros inferiores con presencia de hiperpigmentación y fotosensibilidad. B) Pápulas de Gottron, caracterizados por exantema violáceo sobre articulaciones metacarpofalángicas.
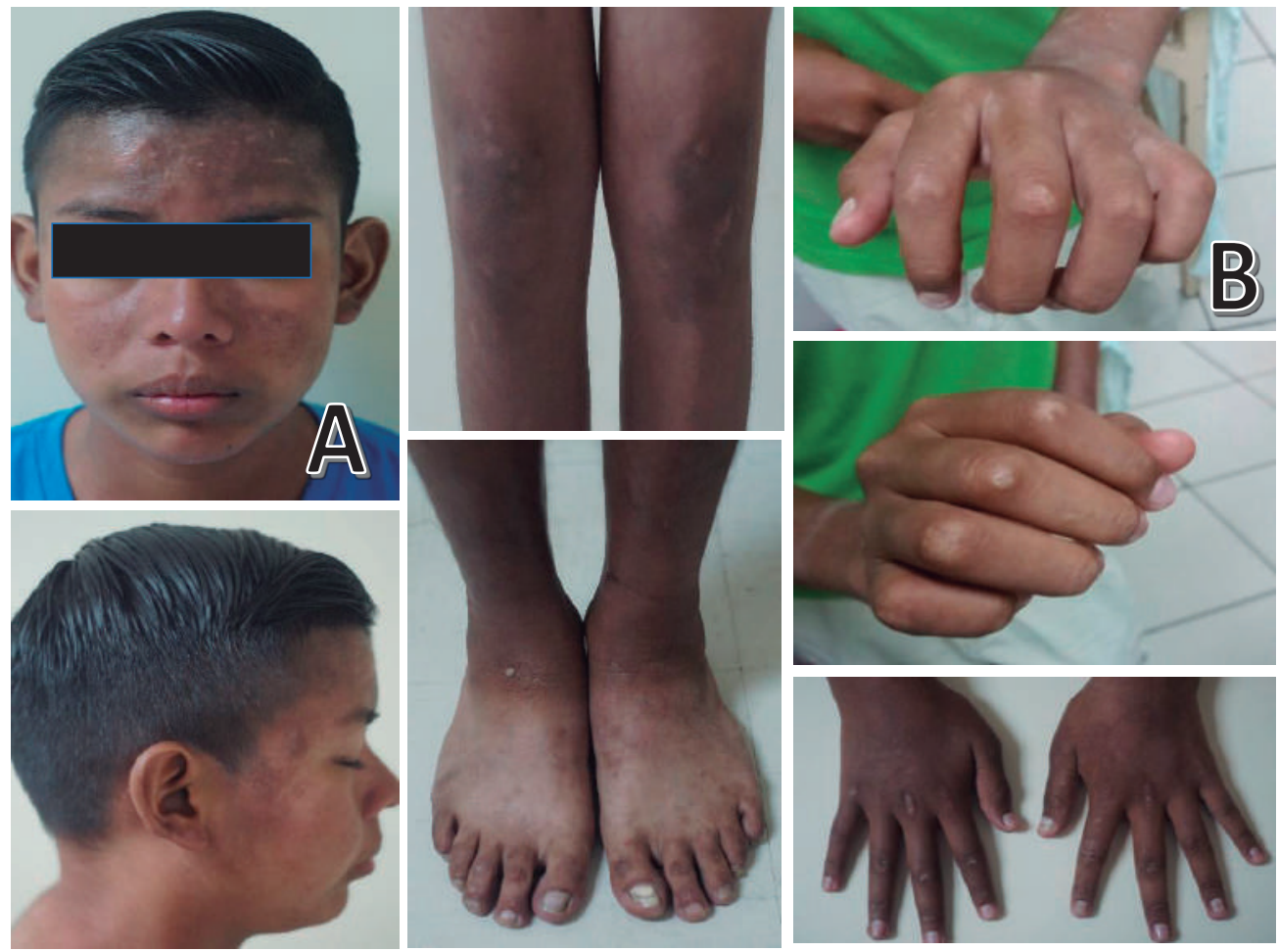

Fuente: Paciente de caso clínico 
Se realizaron exámenes laboratoriales que reportaron: anemia leve, reactantes de fase aguda alterados, presencia de CPK, LDH, TGO aumentadas (Ver Tabla 1).

Tabla No. 1: Hallazgos de laboratorio.

\begin{tabular}{lll}
\hline Hallazgo Laboratorial & Resultado & Valor normal \\
\hline Glóbulos rojos (x 106/uL) & 2.05 & $(4.2-5.4)$ \\
Hemoglobina (g/dL) & 11.6 & $(12-16)$ \\
Hematocrito (\%) & 36 & $(37-47)$ \\
Volumen corpuscular medio & 76.1 & $(82-92)$ \\
(fL) & 23.8 & $(26)$ \\
Hemoglobina corpuscular & 373,000 & $(150-500)$ \\
media (g/dl) & & \\
Plaquetas (x 103/uL) & & \\
Glóbulos blancos (x 10/uL) & 5.9 & $(5.2-12.4)$ \\
Neutrófilos (\%) & 67.8 & $(40-74)$ \\
Linfocitos (\%) & 23.3 & $(35-45)$ \\
Glucosa (mg/dL) & - & $15-37$ \\
BUN (mg/dL) & 6 & $(81-234)$ \\
Creatinina (mg/dL) & 0.45 & $(0.2-1.5)$ \\
TSGO (U/L) & 50 & $(0-40)$ \\
TSGP (U/L) & 45 & $(50-136)$ \\
CPK (U/L) & 439 & $(81-234)$ \\
Lactato Deshidrogenasa & 409 & $(135-145)$ \\
(U/L) & 152 & - \\
Sodio (mEq/L) & Negativo & - \\
Anti DNA & Negativo & - \\
ANA & Negativo & - \\
ANCA & 62 & - \\
Hierro (g/dL) & 101 & - \\
Ferritina (ng/dL) & Negativo & - \\
PCR & 60 & - \\
VES (mm/h) & - & - \\
Anti-Jo-1 & - & - \\
Ac ANTI-SINTETASA & & \\
\hline TSGO: Aspartato-aminotransferasa; BUN: & Nitrógeno \\
\hline & & \\
\hline
\end{tabular}


Se realizaron rayos $\mathrm{x}$ seriadas que demostraron la presencia de calcinosis a nivel de tejidos blandos (Ver Figura 2).

Figura No. 2: Múltiples áreas de calcinosis cutis. A) Radiografía anteroposterior de humero derecho. B) Región articulación humero-radio cubital bilateral. C) Región femoral bilateral. D) Región tibial-peroneal bilateral.
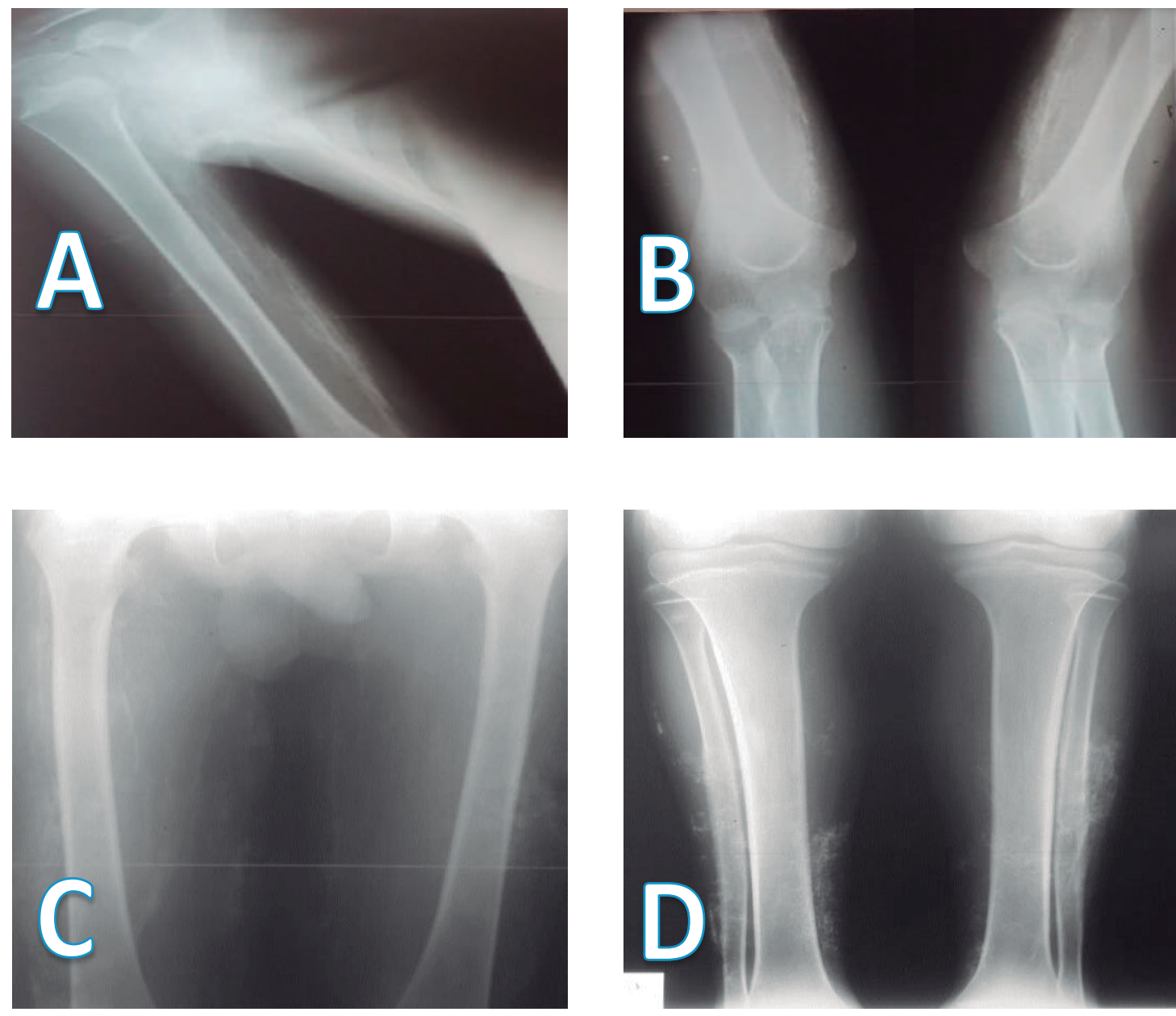

Fuente: foto tomada de expediente clínico.

Asimismo, se realizó estudio de electromiografía que concluyó evidencia de hallazgos compatibles con un proceso miopático generalizado, corroborando diagnóstico de remisión de polimiositis. Se decide realizar estudio anatomo-patológico (biopsia de músculo deltoides) la cual reportó presencia de necrosis, con poca regeneración de fibras musculares, con marcada atrofia perifascicular, las fibras se encuentran redondas en lugar de poligonales.

Marcado infiltrado linfocitico perimisial, datos compatibles con recaída. (Ver Figura $3)$. 
Figura No. 3: Biopsia transversal y longitudinal de músculo deltoides. Secciones longitudinales de músculo deltoides que evidencia áreas de necrosis, 3 focos de infiltrado inflamatorio perimisial y 2 focos de infiltrado perivascular, las fibras se encuentran redondas en lugar de poligonales (Tinción Hematoxilina Eosina (A, B)). En los cortes transversales se observa una marcada atrofia perifascicular que se hace más evidente con la tinción de Tricrómicro de Masson (C) y Acido periódico de Schiff (D).
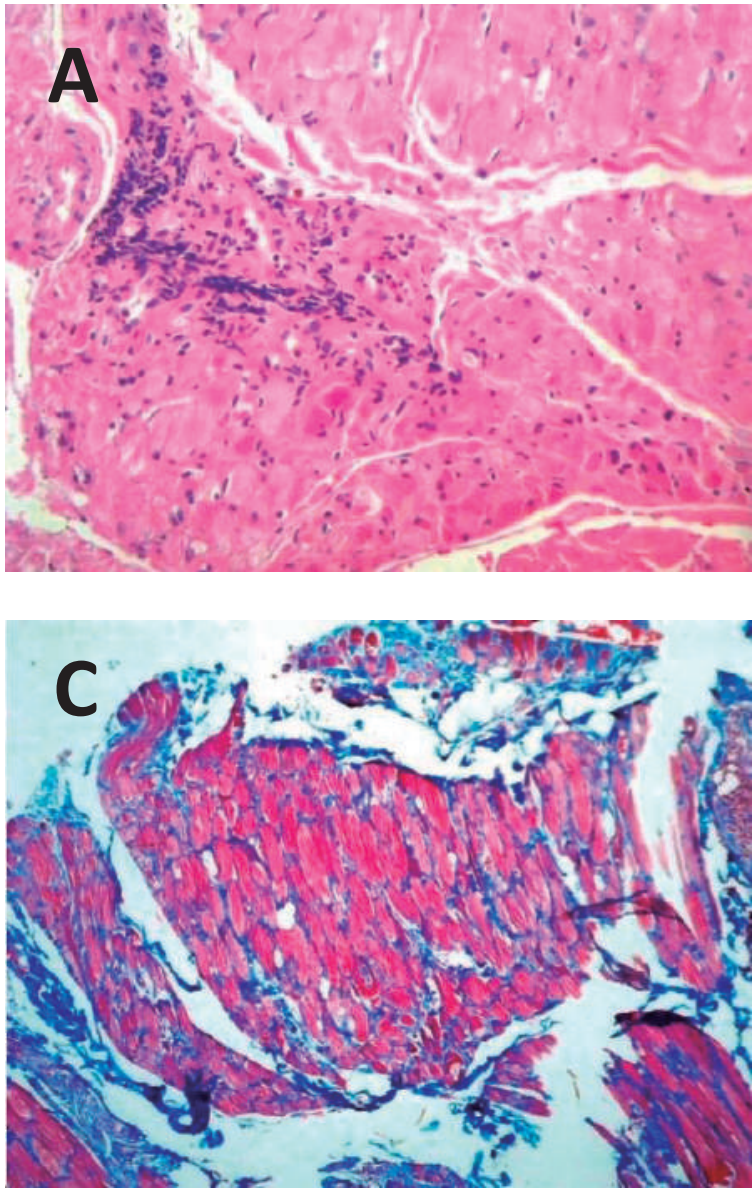

A su vez, se realizó endoscopia digestiva alta que reportó: esofagitis, gastritis, y duodenitis crónica moderada, e hiperplasia con metaplasia intestinal incompleta. El tratamiento consistió en pulsos de metilprednisolona, plaquinol, metotrexate, ácido fólico y administración de inmunoglobulina intravenosa mostrando mejoría evidente de cuadro. Se realiza interconsulta con servicio de reumatología quien decide se omita metotrexate e inicia azatioprina semanal,
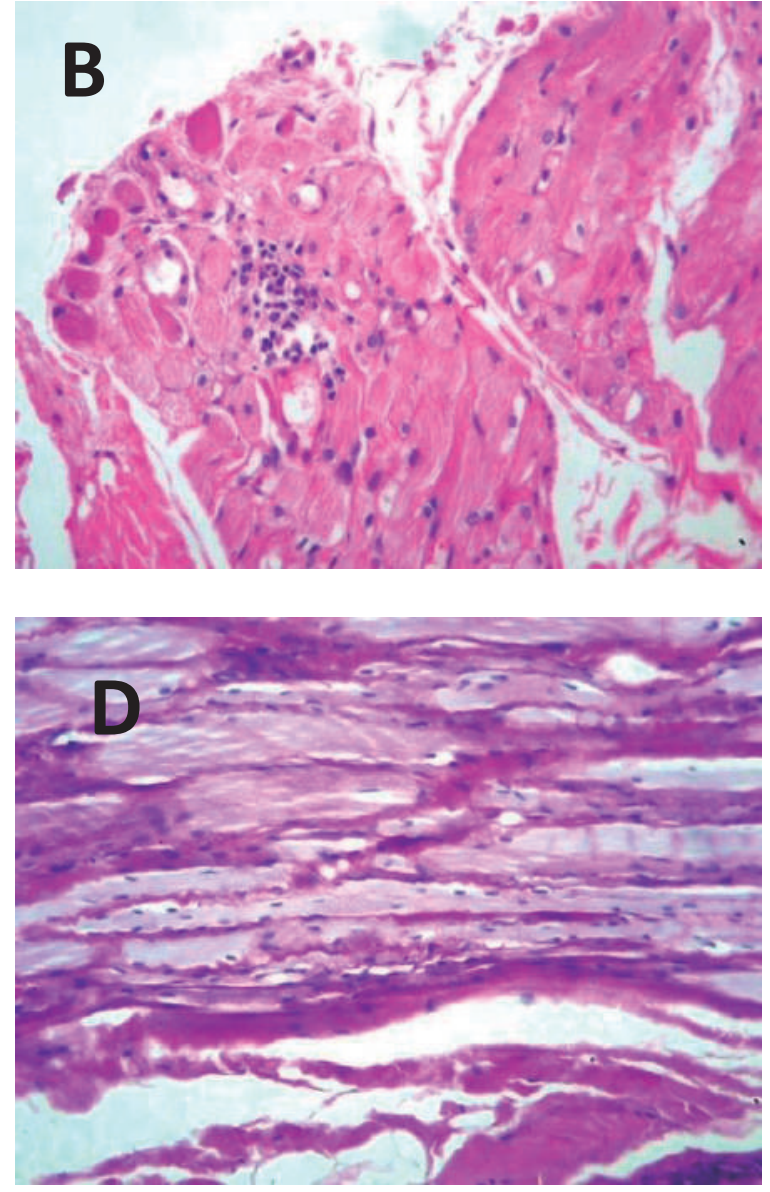

resolucionando cuadro a los 13 días intrahospitalarios.

\section{DISCUSIÓN}

La etiología de las MII es desconocida, se ha sugerido la participación de factores hormonales, infecciosos (virales y bacterianos), ambientales, mecanismos de mimetismo molecular, autoinmunes y genéticos, en la activación y desarrollo de la enfermedad. Entre los posibles factores genéticos impli- 
cados en la patogénesis de la miositis en pacientes caucásicos, se involucra el antígeno leucocitario humano (HLA), particularmente en HLA-DRB1 0301 y DQA1 0501. El principal blanco antigénico en la miopatía idiopática se localiza en el endotelio de los capilares endomisiales. Se ha propuesto que el daño inflamatorio observado en las $\mathrm{Ml}$ es de origen autoinmunitario, con la participación de la inmunidad celular y humoral, debido a la presencia de auto anticuerpos e infiltrados celulares en los tejidos musculares, principalmente compuestos por linfocitos $\mathrm{T}$ y macrófagos, productores de citosinas en polimiositis (PM), así como por linfocitos $B$ productores de anticuerpos en dermatomiositis (DM). ${ }^{(6)}$

Los principales síntomas observados en cohortes retrospectivas de pacientes con MII, incluyen por orden de frecuencia: debilidad $(91 \%)$, mialgias $(43 \%)$, rash $(42 \%)$, disfagia (42\%) y artralgias (36\%); mientras que los hallazgos en el exámen físico fueron: debilidad muscular (87\%), con la siguiente distribución extremidades pélvicas (85\%), extremidades torácicas $(77 \%)$, así como exantema (42\%), rash en heliotropo $(23 \%)$, signo de Gottron (21\%) y signo de chal $(11 \%)$, los cuales concuerdan con las manifestaciones clínicas previas al inicio del diagnóstico y la posterior recaída. ${ }^{6,7)}$ Puede también afectar en algunos casos a otros órganos como el tracto gastrointestinal, corazón, pulmón, riñón y los ojos.

En fases tardías de la enfermedad pueden aparecer otras complicaciones como la lipoatrofia o la calcinosis cutánea, cuya prevalencia ha ido disminuyendo con el tratamiento intensivo precoz. ${ }^{(8)}$ signos y síntomas que concuerdan con la presentación clínica del paciente.
Laboratorialmente la velocidad de sedimentación globular (VSG) suele estar elevada, a pesar de que pueden encontrarse cifras normales, incluso en fases activas. La determinación de la CK es la prueba más útil por su especificidad, ya que es la que refleja con mayor precisión la actividad de la enfermedad. Aunque en general los valores de CK se correlacionan con la actividad clínica, en un $20 \%$ de los casos pueden ser normales.

La determinación de aldolasa sérica, de la lacto-deshidrogenasa (LDH) e incluso de las transaminasas también puede ser útil. Los anticuerpos antinucleares (AAN) son positivos en el $50 \%$ de los casos y el factor reumatoide está presente en el $8 \%$ de los enfermos. Los anticuerpos anti-ADN son siempre negativos. Los anticuerpos que poseen una mayor utilidad diagnóstica son los anti-Jo-1, anticuerpos anti sintetasa que definen a un subgrupo de pacientes con poliartritis, miositis, neumopatía intersticial, fenómeno de Raynaud y "manos de mecánico» (síndrome anti sintetasa). Los anticuerpos anti-Jo-1 se detectan en el 75\% de los casos de MII con afectación pulmonar y solo en el $3 \%$ de los pacientes con miositis sin afectación pulmonar. ${ }^{(9)}$ Datos laboratoriales que concuerdan con los presentados por el paciente.

El diagnóstico de miopatías inflamatorias se sospecha sobre la base de un conjunto de signos y síntomas y es confirmado mediante estudios complementarios, entre los que se incluyen: elevación de enzimas musculares, presencia de autoanticuerpos específicos de miositis, electromiografía con patrón miopático, hallazgos específicos en la biopsia. Los criterios diagnósticos de DM/PM se establecen según Bohan y Peter, los cuales tienen una sensibilidad del $70-100 \%$ y una especificidad del 93\% (Ver Tabla 2). ${ }^{(10,11)}$ 
Tabla No. 2: Criterios de Bohan y Peter para polimiositis y dermatomiositis.

1. Debilidad simétrica y progresiva de la musculatura proximal (Afectación en la mayoría de los casos cintura escapular y pélvica. En casos agudos o de larga evolución puede existir debilidad distal).

2. Biopsia muscular que muestra infiltrado inflamatorio

Presencia de fibras tipo I y II degeneradas y necróticas, junto con un infiltrado inflamatorio formado por linfocitos y macrófagos con algunas células plasmáticas y ocasionalmente PMN en fases iniciales. El infiltrado se localiza perivascular y perifascicular.

\section{Elevación de las enzimas musculares} (Marcadores de lesión muscular: CK, LDH, TGO, TGP y aldolasa.

\section{Alteraciones en el electromiográficas compatibles}

Tríada EMG con evidencia de potenciales de unidad motora polifásicas de amplitud y duración reducidas (miopáticas), fibrilaciones, ondas positivas, irritabilidad insercional y descargas repetitivas de alta frecuencia.

\section{Signos dermatológicos}

Decoloración lila de los párpados (heliotropo) con edema periorbitario y dermatitis eritematosa con descamación preferentemente en las articulaciones metacarpofalángicas e interfalángicas proximales de las manos (signo de Gottron) que también puede afectar a las rodillas, codos, maléolos mediales, cara, cuello y torso superior.

\section{Diagnóstico:}

PM definitiva: 4 de los primeros 4 criterios ( 3 de 4 con rash para DM definitiva).

PM probable: 3 de los 4 primeros criterios (2 de 4 con rash para DM probable).

PM posible: 2 de los 4 primeros criterios (1 de 4 con rash para DM posible).

CK: creatincinasa; LDH: lactatodeshidrogenasa; TGO: Aspartato-aminotransferasa; TGP: Alanino-aminotransferasa; EMG: electromiograma; PM: polimiositis; DM: dermatomiositis.

Los criterios diagnósticos son evidenciados en vista que presenta los 5 criterios, por lo que se hace el diagnóstico definitivo de PDM.

El tratamiento consiste en: 1) Corticoterapia: (tratamiento de primera línea) lo que incluye el uso de prednisona o metilprednisolona. 2) Inmunosupresores: metotrexato, azatioprina, micofenolato mofetilo, ciclosporina, tacrolimús, ciclofosfamida. 3) Antipalúdicos: cloroquina, hidroxicloroquina se proponen en casos de DM con fotosensibilidad e importantes manifestaciones cutá- 
neas. 4) Inmunomoduladores y bioterapias: Inmunoglobulinas intrvenosas. 5) Recambios plasmáticos: plasmaféresis. 6) Anticuerpos monoclonales: Rituximab. 7) Anti-TNF-alfa: etanercept, infliximab. 8) Kinesiterapia: La rehabilitación desempeña, desde luego, un papel importante en la recuperación de la fuerza motora, pero se ha evaluado poco en los estudios. Ningún tratamiento no farmacológico ha demostrado claramente su eficacia en la Ml y, hasta la fecha, sólo la kinesiterapia parece aportar algún beneficio. ${ }^{(12)}$

Las recaídas tienden a ocurrir dentro de los primeros 2 años después de iniciar el tratamiento, así como durante la fase de reducción de los inmunosupresores. El curso crónico, policiclíco o continuo de la enfermedad predicen pobre desenlace en estos pacientes. Los factores pronósticos que afectan la supervivencia de los individuos con miositis incluyen: edad avanzada al inicio de la enfermedad, sexo masculino, tabaquismo, debilidad muscular grave generalizada con disfagia o con afección de músculos de la lengua y faringe, afección cardiovascular, afección pulmonar intersticial de rápida progresión, infecciones, neoplasias.

Las causas más comunes de mortalidad en pacientes con PM/DM son las neoplasias, las infecciones (principalmente neumonía) y la enfermedad pulmonar y cardiovascular. En enfermos con DMJ, la persistencia de las pápulas de Gottron y las alteraciones del lecho ungueal son predictores de retraso en la remisión. ${ }^{(13)}$

Las complicaciones se pueden presentar como propias de la enfermedad: desnutrición secundaria a disfagia persistente e incluso la broncoaspiración, el curso evolutivo puede comportar anquilosis articular con pérdida de la funcionalidad y atrofia muscular. Asimismo, son frecuentes las úlceras cutáneas, vasculitis asociada o calcinosis. A su vez asociadas al tratamiento inmunosupresor: infecciones oportunistas (Pneumocystis jirovecii en el pulmón o Toxoplasma gondii en el sistema nervioso central, micobacterias cutáneas, infecciones víricas por citomegalovirus, virus de Epstein-Barr). ${ }^{(14)}$

Dentro de los diagnósticos diferenciales de PM/DM se encuentran las enfermedades neuromusculares, que cursan con pérdida de fuerza y/o dolor muscular y comprenden los trastornos primarios de motoneurona (superior: esclerosis lateral amiotrófica, enfermedades del asta anterior: atrofias musculares espinales, poliomielitis, y de nervios periféricos: neuropatías), trastornos de la transmisión neuromuscular (miastenia gravis, síndrome de Eaton-Lambert), trastornos primarios del músculo (miopatías inflamatorias idiopáticas, distróficas congénitas, mitocondriales, metabólicas, asociadas a enfermedades endocrinas, por tóxicos y fármacos, infecciosas e inflamatorias asociadas a otras enfermedades (sarcoidosis). ${ }^{(15)}$

Conclusión: La PM y DM en niños tienen un comportamiento clínico algo diferente que en adultos por la presencia de vasculitis; existiendo varios desórdenes que pueden confundirse con esta entidad y retardar su diagnóstico y tratamiento, de ahí que la mayoría de niños tienen un curso prolongado de afección debido a su manejo tardío, con los consiguientes efectos adversos de terapias agresivas que se usan por tiempo prolongado, por lo tanto es muy importante el conocimiento de esta enfermedad en la edad pediátrica y establecer comparaciones con lo reportado en la literatura mundial. 


\section{REFERENCIAS BIBLIOGRÁFICAS}

1. Katsuyuki Shinjo S, Carlos de Souza F.H, Bertacini de Moraes J.C. Dermatomyositis and polymyositis: from immunopathology to immunotherapy (immunobiologics). Rev Bras Reumatol 2013; 53(1):101-110.

2. Selva-O'Callaghan A, Ramos Casals $M$, Grau Junyent J.M. Terapia biológica en pacientes con miopatía inflamatoria idiopática. Med Clin (Barc). 2014; 143(6): 275-280.

3.

Irazoque-Palazuelos F. Barragán-Navarro Y. Epidemiología, etiología y clasificación. Reumatol Clin. 2009;5(S3):2-5.

4. González Crespo M.R. Miopatías inflamatorias idiopáticas. Medicine 2000; 8(30): 1553-1560.

5. Félix Restrepo J. Dermatomiositis-polimiositis. Rev Col Reumatol. 2003;10(2): 135-141.

6. Arellano Valdez C.A, Barrera Cruz A, Cruz Reyes C.V, Jardines de la Luz G, Peralta Juárez R, Sánchez Alonso S. Et al. Diagnóstico y Tratamiento de Polimiositis y Dermatomiositis. México: Secretaría de Salud, 2011.

7. Polo Rodríguez I, Medina Montalvo S, Trasobares Marugán L, Bohórquez Heras C. Manifestaciones cutáneas de las enfermedades reumatológicas. Medicine. 2017; 12(26): 1520-9.

8. Corral-Magaña $\mathrm{O}$, et al. La resonancia magnética muscular y el tratamiento agresivo precoz, claves en la dermato- miositis juvenil. Actas Dermosifiliogr. 2017. http://dx.doi.org/10.1016/j.ad. 2017.07.003.

9. Duró Pujol J.C. Miopatías inflamatorias idiopáticas. Reumatología clínica (primera edición), 2010, pag. 211-217. Disponoble en: https://ac.els-cdn.com/ B9788480866453500159/3-s2.0-B9788 480866453500159-main.pdf?_tid= 1642033f-4fad-428c-be45-f29a1b4aaa5 d\&acdnat=1521745150_633471 fa2e57 ebca1385afc3d713bc15.

10. Bohan A, Peter JB. Polymiositis and dermatomyositis. N Eng J Med 1975; 292: 344-348, 403-407.

11. Dimachkie MM, Barohn RJ, Amato AA. Idiopathic inflammatory myopathies. Neurol Clin. 2014;32(3):595-628.

12. Allenbach $Y$, Benveniste O. Polimiositis, dermatomiositis y otras miopatías inflamatorias idiopáticas. EMC Aparato locomotor. 2015; 48(1): 1-11. Archivo disponible en: http://dx.doi.org/10.1016/S 1286-935X(15)70083-3.

13. Dimachkie MM, Barohn RJ, Amato AA. Idiopathic inflammatory myopathies. Neurol Clin. 2014;32(3): 595-628.

14. González Crespo M. R. Miopatías inflamatorias idiopáticas. Rev Clin Esp 2002; 202(9): 500-508.

15. Turrión Nieves A.I, Martín Holguera R, Sánchez Atrio A.I. Moruno Cruz H. Miopatías inflamatorias idiopáticas. Medicine. 2013 ; 11(33): 2040-7. 